\title{
Efficiency parameters estimation in gemstones cut design using artificial neural networks
}

\author{
Adriano A. Mol a, Luiz S. Martins-Filho ${ }^{\text {b,* }}$, José Demisio S. da Silva ${ }^{c}$, Ronilson Rocha ${ }^{\text {d }}$ \\ ${ }^{a}$ Centro de Estudos em Design de Gemas e Jóias, Univ. do Estado de Minas Gerais, Av. Antonio Carlos 7545, 31270-010 Belo HorizontelMG, Brazil \\ ${ }^{\mathrm{b}}$ Departamento de Computação, Univ. Federal de Ouro Preto, Campus Morro do Cruzeiro, 35400-000 Ouro Preto/MG, Brazil \\ ${ }^{c}$ Lab. Associado de Computação e Matemática Aplicada, Inst. Nacional de Pesquisas Espaciais, Caixa Postal 515, 12227-010 S. José dos Campos/SP, Brazil \\ ${ }^{\mathrm{d}}$ Departamento de Eng. de Controle e Automação, Univ. Federal de Ouro Preto, Campus Morro do Cruzeiro, 35400-000 Ouro Preto/MG, Brazil
}

Received 28 September 2005; received in revised form 23 March 2006; accepted 15 May 2006

\begin{abstract}
This paper deals with the problem of estimating cut results for faceted gemstones. The proposed approach applies artificial neural networks for a faceted gemstones analysis tool that could be further developed for incorporation in a computer-aided-design (CAD) context. Basic concepts concerning gemstone processing are introduced and the design of computational tools using neural networks is discussed. The model presented proposes two criteria to assess the efficiency of lapidary designs for rock crystal quartz: brilliance and yield. Closing the article, 62 different lapidary models were used to train and test the neural network tool.
\end{abstract}

(C) 2006 Elsevier B.V. All rights reserved.

Keywords: Faceted gemstones; Lapidary design; Design efficiency; Artificial neural networks

\section{Introduction}

The minerals qualified as gemstones are remarkable and attractive in consequence of special characteristics like color, brilliance, transparency, hardness, durability and rarity. Its beauty, essentially associated with their optical features, has made these precious stones very attractive for diverse applications related to adornment objects and its rarity has assigned high exchange value to them. Man's fascination with gemstones dates from prehistoric times and since the beginning of civilization, examples of efforts to improve the optical properties of some gemstones are found, aiming at enhancing its beauty and, consequently, its exchange value [1].

One of the most important ways to aggregate value to the gemstones is lapidary, the general denomination of the cutting and polishing processes applied to solid materi-

\footnotetext{
${ }^{*}$ Corresponding author. Tel.: +55 (31) 3559 1663; fax: +55 (31) 3559 1660.

E-mail address: luizm@iceb.ufop.br (L.S. Martins-Filho).
}

als. In the field related to this work, lapidary techniques are still being developed in terms of producing even more sophisticated models, seeking to control color saturation, to maximize the return of incident light from the observer perspective, to obtain interesting distortion effects of light rays inside the gem, to reduce wastage in the raw material cutting, or simply to allow the setting of the gemstone on the jewel assembly $[2,3]$.

This article presents a study of the application of artificial neural networks (ANN) on efficiency in parameters estimation and evaluation of lapidary design results considering the technique of faceting. Application of ANN-based tools on materials science and engineering is becoming an interesting issue for design, characterization and evaluation problems. For instance, some useful ANN applications on materials science can be found in $[5,4,6]$. The results or efficiency of such lapidary designs can be measured in terms of optical performance and weight retention. The optical performance of gemstones can be judged primarily for its brilliance, defined as the portion of incident light that returns to an observer after traveling through different paths inside 
the gem. The weight retention, here called yield, is the weight ratio between the raw material and the final faceted gem obtained after the cutting and polishing tasks [7-9]. Computational tools based on artificial neural networks have been applied to different kinds of problems where it is necessary to extract knowledge from strongly nonlinear and complex systems, being the case of the relationship between the geometry of three-dimensional faceted gem and its brilliance and yield results.

This study's subject relevance can be observed in some recent related works concerning these two main gems properties. For instance, an interesting hybrid artificial intelligence approach for the yield improvement is described in [10]. A computational tool for quality grading, based on rule-based knowledge representation, fuzzy logic and genetic algorithms, provides better understanding of lapidary possibilities and therefore can contribute to wastage reduction when real faceting actually takes place. Another innovative work presents a detailed study of optical effects inside a polyhedral faceted gem, modeling the complex behavior of light in such materials, and proposes an efficient computer graphics algorithm for rendering faceted gemstones and obtaining a photo-realistic graphic representation of optical effects such as color and brightness [11].

The international jewellery industry is the final destination of most part of faceted gems, turning about 14 billion dollars per year on the international trade (estimative of

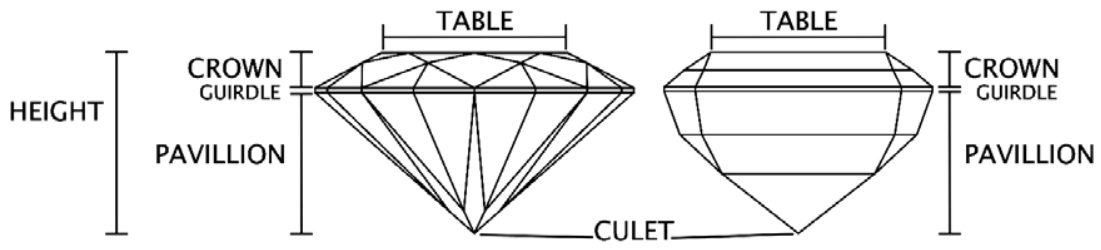

Fig. 1. Nomenclature of a faceted gemstone.

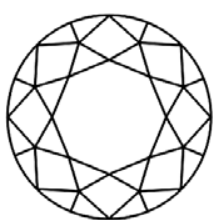

(a)

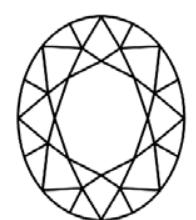

(b)

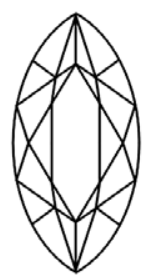

(c)

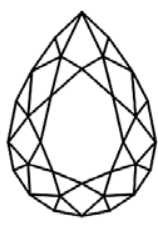

(d)

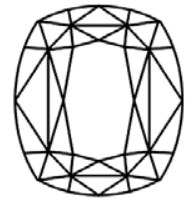

(e)

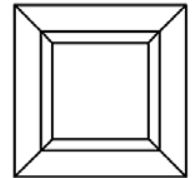

(f)

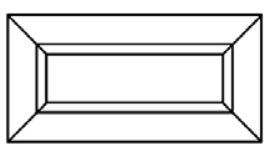

(g)

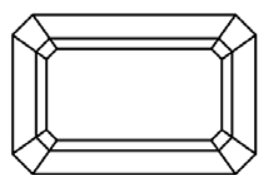

(h)

Fig. 2. Top view of typical gemstones: (a) round, (b) oval, (c) navette or marquise, (d) pear, (e) cushion, (f) square, (g) rectangular or baguette, (h) octagonal or emerald cut.

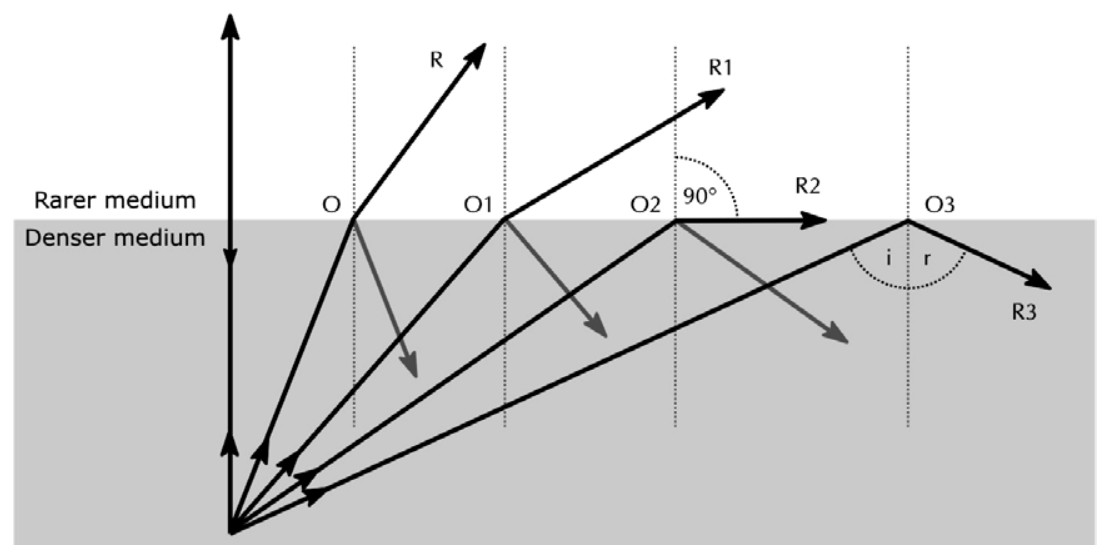

Fig. 3. Light refraction and total internal reflection phenomena on two different materials interface, with different refraction indexes. 
Brazilian Institute of Precious Gemstones and Metals IBGM [12]). Developing countries such as Brazil, the major producer of rough gemstones, could improve economical turnovers with better utilization of available mineral resources. It can be verified in the European jewellery report of the Centre for the Promotion of Imports from Developing Countries - CBI [13]. The lapidary taken as an industrial design process shows ways to aggregate value to mineral production and to improve this relevant sector. The Brazilian case of under-exploitation of its actual economic potential constitutes the main motivation for this study [14].

\section{Lapidary}

The lapidary as procedure to process minerals for adornment purpose has its origins, according to historians, on the region of Iraq in 5th AC century. The early lapidary designs were obtained by polishing natural faces of crystals and pebbles found on alluvium deposits. Later appears the cabochon model, consisting of domed polished gemstones without facets, with smooth, rounded edges. Around the 13th DC century, better optical results are obtained with faceted lapidary models, produced using flat discs sprayed with powdered diamond and corundum to create small cuts by abrasion. The shapes became prismatic to enhance the already known significant effects like brilliance and light dispersion. In the 20th century, lapidary designs for the diamond industry begin to be described by well-defined parameters, and started the ongoing research about the improvement of these models.

The basic nomenclature of a faceted model is shown in Fig. 1. The model parameters are related to dimension proportions between the indicated elements. The cutting models can present diverse characteristics in terms of shape. Fig. 2 shows some typical geometry shapes.

Analyzing the interaction of the light with a transparent gem, it is possible to remark the main physical phenomena

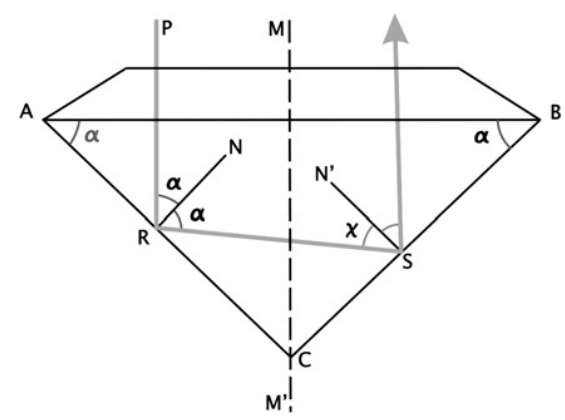

Fig. 4. Light ray path inside section of faceted gem.
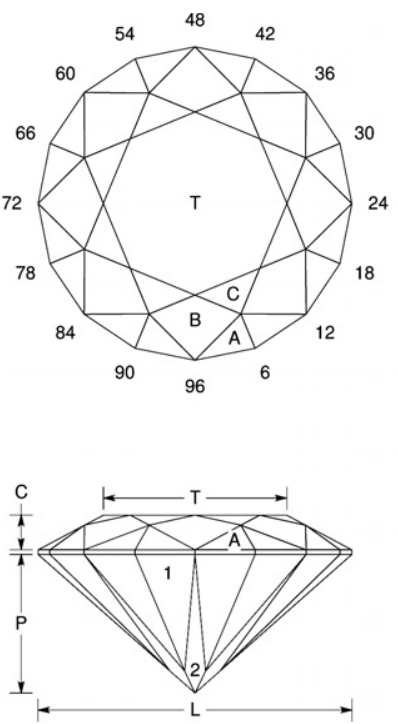

PAVILION

G $90.00^{\circ}$ 03-09-15-2127-33-39-45$51-57-63-69-$ $75-81-87-93$

$142.50^{\circ} 03-09-15-21$ 27-33-39-4551-57-63-6975-81-87-93

$241.50^{\circ} 96-12-24-36$ 48-60-72-84
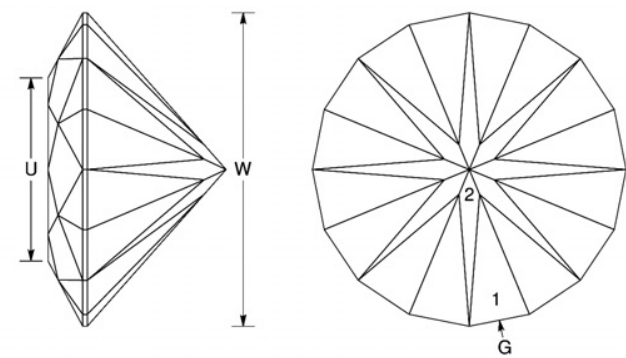

Standard Round Brilliant

GemCad for Windows User's Guide

Angles for R.I. $=1.540$

$57+16$ girdles $=73$ facets

8 -fold, mirror-image symmetry

96 index

$\mathrm{L} / \mathrm{W}=1.000 \mathrm{~T} / \mathrm{W}=0.584 \mathrm{U} / \mathrm{W}=0.584$

$\mathrm{P} / \mathrm{W}=0.442 \mathrm{C} / \mathrm{W}=0.111$

Vol. $/ \mathrm{W}^{3}=0.182$

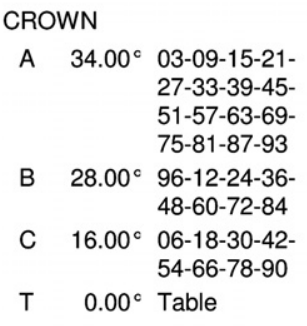

CROWN

C:IArquivos de programas\GemCadWin\DesignsiSRBISRB.gem

Fig. 5. A lapidary model diagram (standard round brilliant). 
in terms of geometric optics. When an incident light ray reaches the gem surface, part of it is reflected and the other part is transmitted or refracted across the air-gem interface. The refracted fraction of the ray travels inside the gem and so it repeats this phenomenon when it reaches another gem-air interface, at an internal surface this time. Fig. 3 illustrates this optical phenomenon, where it is shown that there exists a certain angle value that delimitates the occurrence of refraction; this angle value is called critical angle. The Snell law gives the relation between the incident angle and refraction angle:

$\frac{c}{v}=n=\frac{\sin i}{\sin r}$

where $c$ is light velocity in the air, $v$ is the light velocity inside the gem, $n$ is the refraction index of the gem material, $\sin (i)$ is the sinus of incident angle, and $\sin (r)$ is the sinus of refraction angle.

Together with table size, the angles formed by the gem crown and the pavilion, with reference to the horizon plan (the girdle), define the geometry and are determinant of the brilliance criterion value, considering the refraction index of the specific gem material. Fig. 4 shows one incident angle being totally reflected back to observer eye after incising at an angle equal to pavilion angle $\alpha$ and being reflected at an angle $\chi$. The pavilion angles of a gemstone should therefore be larger than the critical angle for each refraction index.

The brilliance, an essential property for a gem evaluation, is defined as the fraction of the incident light that returns to an external observer positioned frontally to the crown, after successive internal reflections.

Another main feature of a gem-cutting model is the yield, i.e., the weight ratio between uncut rough stone and finished faceted gem. In general, the raw material is presented to the lapidary processing in strongly irregular and diverse shapes. Consequently, for comparative criterion, a convention is established as a percent volume of the faceted gem inside a cube of the smallest size that may contain this gem.

\section{Description of the model}

A cutting model can be defined through a diagram with the description of all geometric parameters, dimension ratios, quantity of facets, and necessary angles for each facet. Fig. 5 shows an example of a cutting diagram for a round standard brilliant generated by GemCAD software. This specialized software provides an estimation of brilliance and material utilization for the models considered in this article. Its estimation procedure is based on inverse ray tracing considering the geometry and refraction index

Table 1

Design parameters of studied lapidary models (first part)

\begin{tabular}{|c|c|c|c|c|c|c|c|c|c|}
\hline & Model & Facets & $T / W$ & $T / L$ & $P / W$ & $C / W$ & $H / W$ & Yield & Brilliance \\
\hline 1 & 2-6-3 Triangle & 52 & 0.516 & 0.490 & 0.380 & 0.188 & 0.588 & 0.187 & 75.4 \\
\hline 2 & 3 Corner triangle & 46 & 0.595 & 0.573 & 0.418 & 0.188 & 0.626 & 0.230 & 79.2 \\
\hline 3 & 6 Star & 79 & 0.474 & 0.457 & 0.462 & 0.209 & 0.691 & 0.254 & 72.0 \\
\hline 4 & 8 Round & 57 & 0.490 & 0.490 & 0.435 & 0.168 & 0.623 & 0.226 & 78.5 \\
\hline 5 & 9 By check round & 33 & 0.268 & 0.268 & 0.435 & 0.209 & 0.664 & 0.224 & 92.8 \\
\hline 6 & Acorn tri & 70 & 0.532 & 0.496 & 0.457 & 0.166 & 0.643 & 0.223 & 74.0 \\
\hline 7 & Beginner check & 27 & 0.267 & 0.267 & 0.435 & 0.191 & 0.646 & 0.216 & 90.2 \\
\hline 8 & Brilliant marquise & 59 & 0.753 & 0.537 & 0.437 & 0.141 & 0.598 & 0.243 & 84.6 \\
\hline 9 & $\mathrm{Bzzz}$ & 101 & 0.482 & 0.482 & 0.481 & 0.175 & 0.676 & 0.251 & 70.0 \\
\hline 10 & China check & 25 & 0.442 & 0.442 & 0.435 & 0.169 & 0.624 & 0.285 & 82.6 \\
\hline 11 & Circus tent & 105 & 0.392 & 0.392 & 0.440 & 0.259 & 0.719 & 0.241 & 70.0 \\
\hline 12 & Crazy triangle & 18 & 0.000 & 0.000 & 0.426 & 0.123 & 0.569 & 0.145 & 76.1 \\
\hline 13 & Cross point & 49 & 0.468 & 0.468 & 0.445 & 0.212 & 0.677 & 0.246 & 70.7 \\
\hline 14 & Cross & 47 & 0.544 & 0.409 & 0.511 & 0.248 & 0.779 & 0.428 & 70.6 \\
\hline 15 & Cut corner crazy tri & 24 & 0.000 & 0.000 & 0.453 & 0.190 & 0.663 & 0.188 & 80.7 \\
\hline 16 & d Qtz round & 97 & 0.461 & 0.461 & 0.549 & 0.228 & 0.797 & 0.316 & 72.6 \\
\hline 17 & Dagger bottom & 41 & 0.393 & 0.393 & 0.483 & 0.214 & 0.718 & 0.315 & 74.4 \\
\hline 18 & Double cross square & 73 & 0.594 & 0.594 & 0.529 & 0.212 & 0.761 & 0.363 & 75.0 \\
\hline 19 & Easy tri & 31 & 0.536 & 0.535 & 0.399 & 0.175 & 0.593 & 0.187 & 83.7 \\
\hline 20 & Fancy & 59 & 0.568 & 0.473 & 0.479 & 0.226 & 0.726 & 0.373 & 82.8 \\
\hline 21 & Fanned triangle & 52 & 0.303 & 0.295 & 0.392 & 0.213 & 0.625 & 0.211 & 88.3 \\
\hline 22 & Frosted star & 79 & 0.533 & 0.514 & 0.459 & 0.182 & 0.661 & 0.251 & 60.0 \\
\hline 23 & Harlequin square & 41 & 0.400 & 0.400 & 0.488 & 0.208 & 0.717 & 0.319 & 70.0 \\
\hline 24 & Hopes square & 53 & 0.505 & 0.505 & 0.460 & 0.226 & 0.706 & 0.279 & 75.0 \\
\hline 25 & Keely cisor & 37 & 0.833 & 0.626 & 0.466 & 0.131 & 0.618 & 0.440 & 84.0 \\
\hline 26 & Light star & 169 & 0.472 & 0.472 & 0.449 & 0.192 & 0.661 & 0.226 & 63.5 \\
\hline 27 & Malt squared & 53 & 0.463 & 0.463 & 0.479 & 0.232 & 0.731 & 0.306 & 72.6 \\
\hline 28 & Mask rectangle & 61 & 0.900 & 0.642 & 0.596 & 0.259 & 0.875 & 0.545 & 70.0 \\
\hline 29 & Mock emerald & 33 & 0.884 & 0.708 & 0.499 & 0.143 & 0.662 & 0.409 & 82.7 \\
\hline 30 & North star & 137 & 0.459 & 0.459 & 0.437 & 0.181 & 0.638 & 0.215 & 75.0 \\
\hline 31 & np Point & 67 & 0.542 & 0.534 & 0.430 & 0.227 & 0.677 & 0.253 & 73.6 \\
\hline
\end{tabular}


of a specific gem-cutting model. Although considered fairly simple, compared to very sophisticated models such as described in [7,11], its results show similar relations between proportional variations and brilliance in macroanalysis. To provide the models for the study, designs for quartz were collected at [15], a commercial website that presented an organized and easily accessible collection. The GemCAD outputs for brilliance and yield will be used to evaluate the results obtained by the proposed neural network based tool.

According to this study assumptions concerning the faceted gemstone evaluation, two properties are preponderant: brilliance and yield. The gem design parameters, supposedly determinant of these evaluation aspects, can be enumerated: (i) total number of gem facets $F$; (ii) dimension ratio between table and width $T / W$; (iii) dimension ratio between table and length $T / L$; (iv) dimension ratio between pavilion and width $P / W$; (v) dimension ratio between crown and width $C / W$; (vi) dimension ratio between total height (crown height + girdle thickness + pavilion depth) and width $H$ / $W[7,16]$. Remark: for round models like this one showed in Fig. 5, $W=L$ and, consequently, $T / W=T / L$. Tables 1 and 2 present the design parameters values for the studied gem models.

Even if not exhaustive, given the diversity of faceting possibilities, the lapidary diagrams studied comprise vari- ous shapes and geometric features to ensure a representative faceted gemstones assortment. Some examples of the considered models are shown in Fig. 6.

\section{Parameters estimation}

The proposed tool for faceted gemstones evaluation is based on artificial neural networks, which are intrinsically parallel and distributed computation systems inspired on the biological brain functions. These systems associate input and output data through artificial neuron units, or nodes, each of which performs a linear combination of the received signals and sends the result to an activation function used to simulate the firing process of a biological neuron. They are organized in layers from which they connect to each of the neurons in the subsequent layer, thus leading to a neural network. Weights values are assigned to the connections among the neurons in the different layers. A general scheme of a feed-forward ANN is presented in Fig. 7 in which the signals propagate from the input to the output without any kind of signal feedback.

A mathematical model of the artificial neuron function was initially proposed by neuro-physiologist Warren McCulloch and mathematician Walter Pitts, in 1943 [18]. In this model, the neuron output is activated by the linear combination of the inputs (cf. Fig. 8). It can be considered

Table 2

Design parameters of studied lapidary models (second part)

\begin{tabular}{|c|c|c|c|c|c|c|c|c|c|}
\hline & Model & Facets & $T / W$ & $T / L$ & $P / W$ & $C / W$ & $H / W$ & Yield & Brilliance \\
\hline 32 & op Blade & 77 & 0.482 & 0.482 & 0.437 & 0.150 & 0.607 & 0.202 & 82.1 \\
\hline 33 & Point square check & 24 & 0.000 & 0.000 & 0.435 & 0.225 & 0.679 & 0.292 & 88.5 \\
\hline 34 & Astor star & 49 & 0.472 & 0.472 & 0.537 & 0.202 & 0.759 & 0.339 & 89.2 \\
\hline 35 & Diagonal 1.5 & 15 & 0.856 & 0.571 & 0.500 & 0.338 & 0.858 & 0.767 & 89.4 \\
\hline 36 & Glass triangle & 67 & 0.433 & 0.432 & 0.447 & 0.198 & 0.666 & 0.202 & 80.0 \\
\hline 37 & Diamond perception & 25 & 0.347 & 0.261 & 0.361 & 0.141 & 0.522 & 0.167 & 84.6 \\
\hline 38 & Prop & 25 & 0.351 & 0.351 & 0.458 & 0.173 & 0.651 & 0.256 & 93.2 \\
\hline 39 & Quartz shield & 44 & 0.507 & 0.507 & 0.426 & 0.191 & 0.637 & 0.232 & 82.8 \\
\hline 40 & z Diamonds & 13 & 0.892 & 0.595 & 0.500 & 0.254 & 0.774 & 0.651 & 90.7 \\
\hline 41 & Scot plaid & 97 & 0.511 & 0.442 & 0.469 & 0.202 & 0.691 & 0.271 & 69.2 \\
\hline 42 & Simply x'ed & 25 & 0.541 & 0.541 & 0.488 & 0.207 & 0.714 & 0.324 & 70.0 \\
\hline 43 & Spin v & 37 & 0.458 & 0.397 & 0.475 & 0.276 & 0.771 & 0.297 & 65.0 \\
\hline 44 & Starred & 71 & 0.471 & 0.471 & 0.428 & 0.176 & 0.624 & 0.199 & 89.8 \\
\hline 45 & Step up & 49 & 0.493 & 0.493 & 0.452 & 0.153 & 0.625 & 0.249 & 80.0 \\
\hline 46 & Straight pedals & 55 & 0.566 & 0.490 & 0.501 & 0.243 & 0.764 & 0.306 & 76.0 \\
\hline 47 & Strip o & 59 & 0.614 & 0.512 & 0.485 & 0.236 & 0.741 & 0.343 & 74.0 \\
\hline 48 & Wink & 51 & 0.768 & 0.578 & 0.485 & 0.215 & 0.720 & 0.353 & 78.2 \\
\hline 49 & Squeezed square & 20 & 0.000 & 0.000 & 0.458 & 0.144 & 0.622 & 0.241 & 94.6 \\
\hline 50 & Square ziggie & 14 & 0.000 & 0.000 & 0.466 & 0.144 & 0.630 & 0.336 & 86.4 \\
\hline 51 & Mock check squares & 17 & 0.807 & 0.807 & 0.466 & 0.239 & 0.726 & 0.372 & 90.3 \\
\hline 52 & Two spin star & 30 & 0.000 & 0.000 & 0.450 & 0.164 & 0.635 & 0.215 & 92.8 \\
\hline 53 & Glider & 12 & 0.913 & 0.489 & 0.431 & 0.127 & 0.578 & 0.257 & 81.3 \\
\hline 54 & Huntress & 43 & 0.457 & 0.443 & 0.447 & 0.164 & 0.631 & 0.222 & 87.9 \\
\hline 55 & Kiss & 12 & 0.000 & 0.000 & 0.459 & 0.230 & 0.709 & 0.174 & 88.6 \\
\hline 56 & Low boy & 45 & 0.713 & 0.713 & 0.454 & 0.129 & 0.604 & 0.202 & 75.8 \\
\hline 57 & Spike & 19 & 0.305 & 0.305 & 0.307 & 0.106 & 0.434 & 0.092 & 93.1 \\
\hline 58 & Spiro & 46 & 0.384 & 0.357 & 0.441 & 0.193 & 0.654 & 0.239 & 86.9 \\
\hline 59 & Tess's pentagram & 23 & 0.820 & 0.640 & 0.396 & 0.175 & 0.591 & 0.256 & 82.6 \\
\hline 60 & Triga light house & 58 & 0.509 & 0.492 & 0.420 & 0.194 & 0.633 & 0.204 & 80.2 \\
\hline 61 & Thorn & 17 & 0.840 & 0.674 & 0.383 & 0.130 & 0.532 & 0.179 & 91.2 \\
\hline 62 & Zip & 15 & 0.656 & 0.656 & 0.462 & 0.099 & 0.581 & 0.220 & 89.4 \\
\hline
\end{tabular}


07

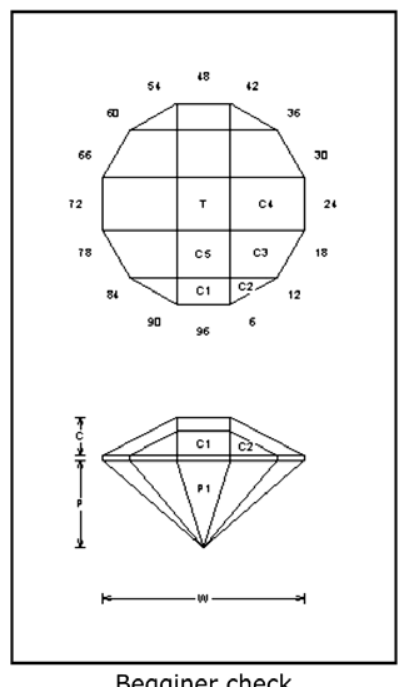

23

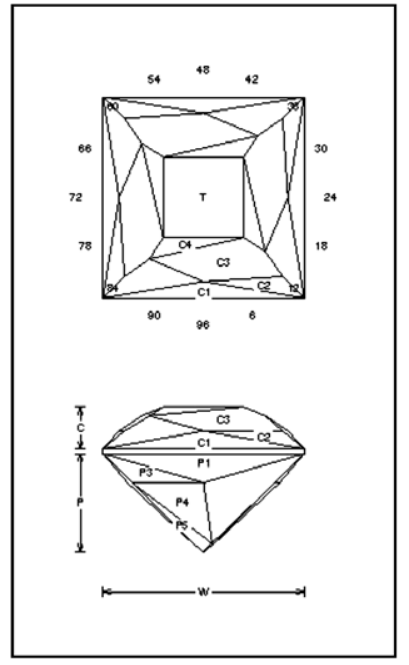

Harlequin square
14

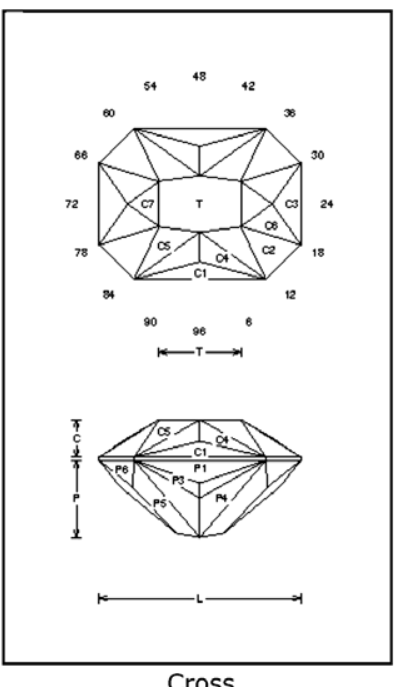

12

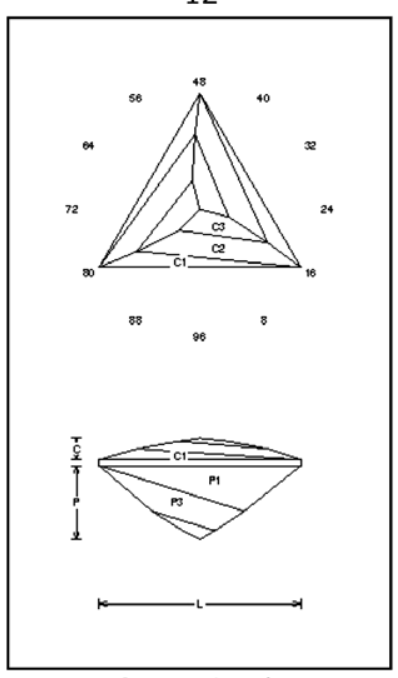

Crazy triangle
08

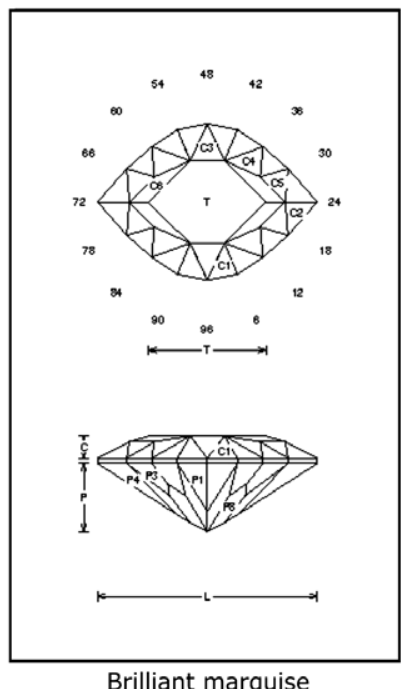

44

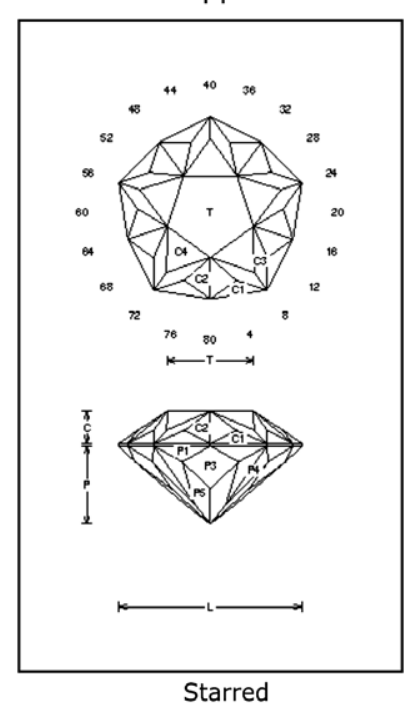

Fig. 6. Some lapidary models studied in this work.

as a transfer function producing the output value as a function of the weighted inputs. Different mathematical functions may be used for the neuron activation.

The main concern in artificial neural networks is the assignment of appropriate weights to the connections. In 1958, based on the works of McCulloch and Pitts and of neuro-physiologist Donald Hebb, who first postulated a learning paradigm for the biological neurons, Frank Rosenblatt proposed a learning algorithm called the perceptron neural network, which in its simplest architecture is composed of a McCulloch and Pitts neuron with several inputs. This algorithm gradually finds the appropriate weights by presenting input-output pairs to the network, that is, the procedure searches for the extraction of input-output relation. When the training procedure is accomplished for an appropriate choice of training data set, the ANN is able to estimate output variables for unknown input data. However, it may only solve classification problems of linearly separated classes.

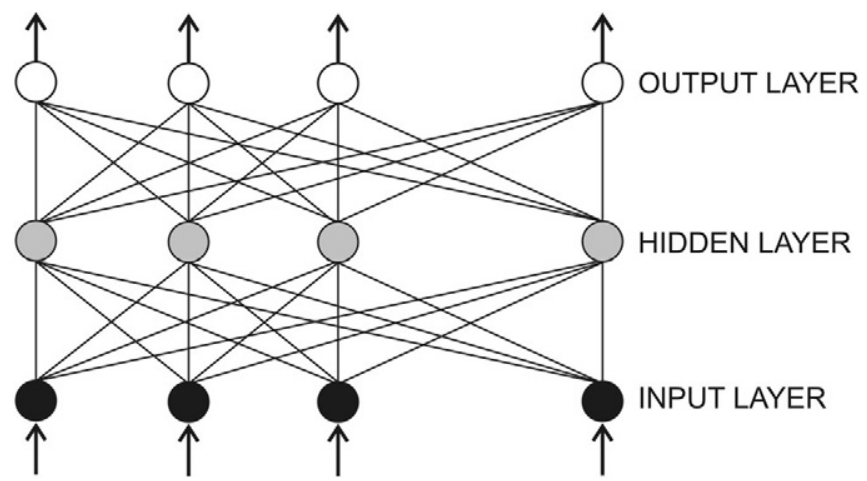

Fig. 7. Scheme of a simple feed-forward ANN. 


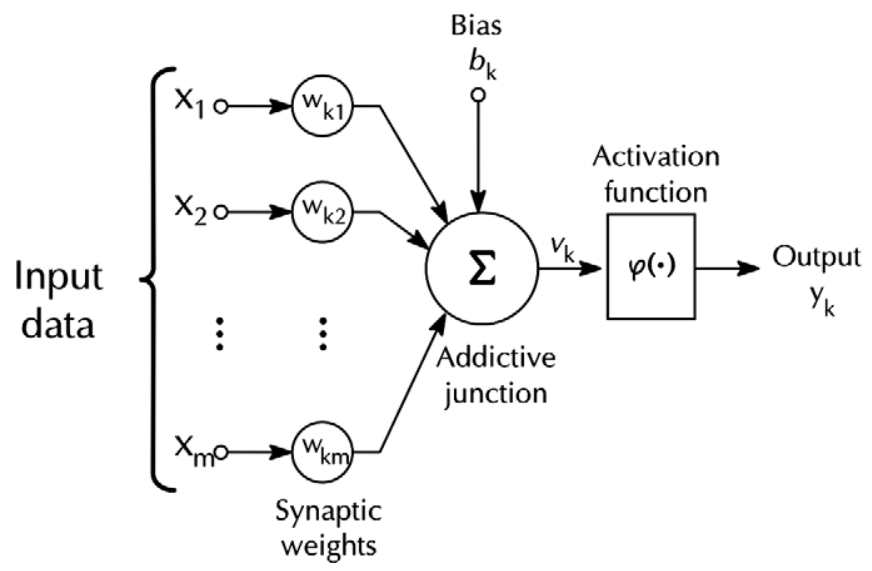

Fig. 8. A mathematical model of a computational neuron.

Several subsequent connected layers of perceptrons lead to the multilayer perceptron architecture as in Fig. 7. That is, in addition to the input and output layers, the multilayer architecture may have a number of intermediate layers, called hidden layers. The use of nonlinear activation functions in the neurons required the development of a different learning strategy to approach nonlinear problems. A training procedure for the multilayer perceptron was proposed by Rumelhart in 1986, based on the back-propagation of the error signals computed at the neural network outputs. The algorithm consists of estimating the local gradient of each neuron in each layer to proportionally adjust the weights.

In the learning process, input and output data are organized in vectors. The number of neurons in the input and output layers depend on the input and output vectors dimensions, respectively. The estimation of the number of hidden neurons, however, is an unsolved issue and usually it is achieved by experimenting different neural network architectures and choosing the one that solves the problem requiring less computation resources.

In this training algorithm, when a new input vector $i$ is chosen from the training data and it is presented to the neural network input, an output vector $o_{\mathrm{a}}$ results and it is compared to the desired (and known) output vector $o_{\mathrm{d}}$. A possible description of the network error may be given by the squared difference:

$e=\left(o_{\mathrm{d}}-o_{\mathrm{a}}\right)^{\mathrm{T}} \cdot\left(o_{\mathrm{d}}-o_{\mathrm{a}}\right)$

The error is a measure of how close the ANN result is from the desired value for a particular input. The purpose of the backpropagation algorithm is to minimize the sum of squared errors for the entire training data (the mean square error - mse). This problem can be described by the minimization of an objective function:

mse $=\frac{1}{N} \sum_{n=1}^{N} e_{n}=\frac{1}{N} \sum_{n=1}^{N}\left(o_{\mathrm{d}(n)}-o_{\mathrm{a}(n)}\right)^{\mathrm{T}} \cdot\left(o_{\mathrm{d}(n)}-o_{\mathrm{a}(n)}\right)$

The backpropagation algorithm is an interactive resolution approach for this optimization problem. The numerical resolution may require many cycles of presenting the training data and controlling the objective function decrease until it reaches a target value. Many computational packages of numerical calculus offer toolboxes implementing different training algorithms based on different optimization strategies.

The main step of the algorithm is the weight adjustment process performed after calculation of the local gradient of the neuron $k$, as it is described in [18]. A weight $w_{k i}$ from the $i$ th input to neuron $k$ is updated as

$w_{k i}=w_{k i}+\eta \times \delta_{k} \times y_{i}$
04

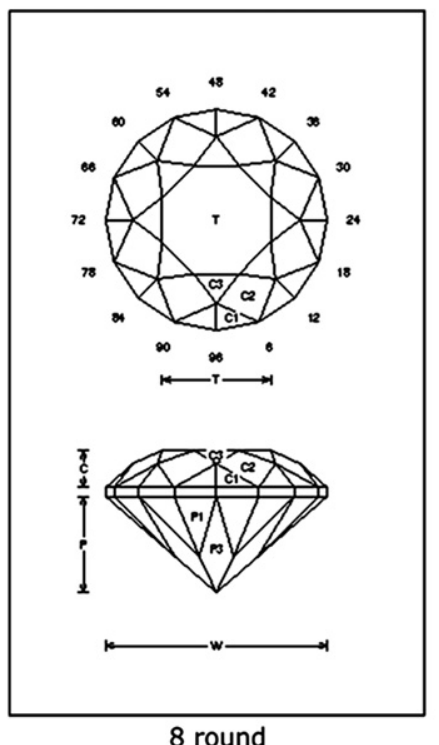

13

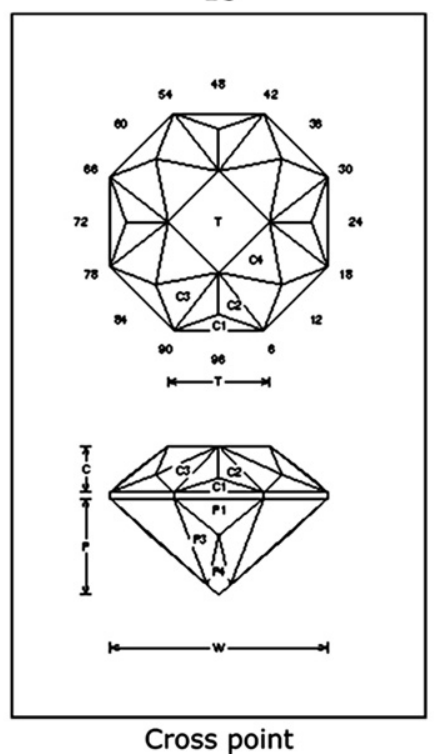

58

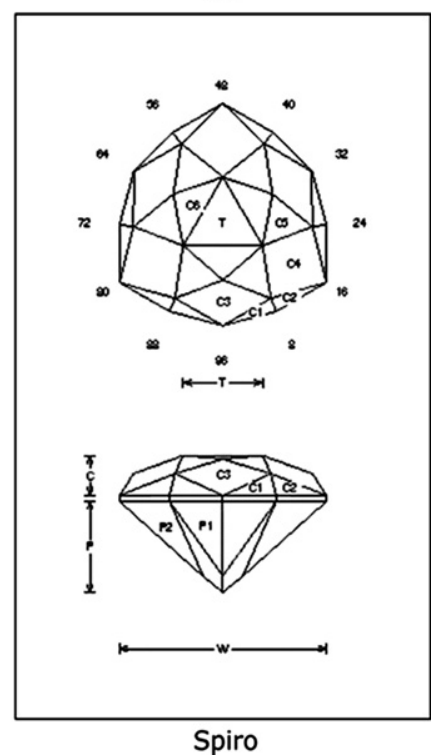

Fig. 9. Lapidary models used for testing the trained ANN. 
where $\eta$ is the learning rate; $\delta_{k}$ is the local gradient of neuron $k$; and $y_{i}$ is the input flowing through weight $w_{k i}$.

The design of ANN architecture for a specific application requires the choice of the network type choice, the number of layers, the input and output variables, and the mathematical activation function of each neuron. Furthermore, it is necessary to collect relevant data relative to the problem. In this paper, an ANN based solution is pursued for the problem of lapidary design. The considered data for the rock crystal quartz is composed by the 62 faceting diagrams set discussed in Section 2.

In the network training procedure, the faceting diagrams were divided in two groups: a training group with 59 models, and a second group with three models for testing the resulting network. The models of testing group were chosen arbitrarily: numbers 4, 13 and 58, see Fig. 9. The input vectors were normalized and scaled (values between 0 and 1), and the number of neurons for each layer was defined by considering practical hints based on several ANN applications described in [18].

The proposed neural network architecture was designed as a multilayer perceptron composed by an input layer with 6 nodes, one hidden layer with 12 nodes, and the output layer with 2 nodes corresponding to the brilliance and yield attributes. The activation function was chosen as the logistic function with output in the closed interval $(0,1)$ [17-19]. This function is monotonically increasing and smooth, which are essential features for the backpropagation algorithm:

$y=\frac{1}{1+e^{-x}}$

Other ANN parameters are the learning rate and the target error. In the present implementation an adaptive learning rate approach was chosen while the target error was established as $10^{-3}$, based on the project choices.

\section{Results}

The training process was followed by the test, where the trained ANN-based tool was applied to analyze the testing data set, composed of models numbers 4 (eight round), 13 (cross point), and $\mathbf{5 8}$ (spiro). This procedure was repeated several times because the training algorithm uses random numbers generator to initialize the network state (the values of biases and connections' weights). Table 3 shows the results for 45 training and test procedure: the error average for the six estimated parameters values (brilliance and yield of three models), the maximum value between the six parameters errors, and final value of the training performance index after 5000 epochs. For these 45 repetitions, the average values of mean and maximum errors obtained are $4.62 \%$ and $9.74 \%$, respectively.

The details of one of these training and test procedures, the results of each parameter estimation and its known values (brilliance and yield) for the three test group models, and the respective errors can be seen in Table 4, and the
Table 3

Results of training and tests procedure: mean and maximum error, and training performance index

\begin{tabular}{|c|c|c|c|}
\hline $\begin{array}{l}\text { Training } \\
\text { and test }\end{array}$ & $\begin{array}{l}\text { Mean estimation } \\
\text { error }(\%)\end{array}$ & $\begin{array}{l}\text { Max estimation } \\
\text { error }(\%)\end{array}$ & $\begin{array}{l}\text { Performance } \\
\text { index }\end{array}$ \\
\hline 1 & 4.78 & 9.35 & 1.92 \\
\hline 2 & 6.45 & 12.21 & 1.49 \\
\hline 3 & 6.24 & 9.73 & 1.32 \\
\hline 4 & 5.32 & 12.97 & 1.44 \\
\hline 5 & 4.58 & 7.82 & 1.79 \\
\hline 6 & 5.86 & 11.61 & 1.78 \\
\hline 7 & 3.80 & 7.82 & 1.65 \\
\hline 8 & 4.61 & 8.99 & 2.06 \\
\hline 9 & 4.37 & 8.52 & 1.17 \\
\hline 10 & 3.39 & 7.91 & 1.76 \\
\hline 11 & 3.95 & 6.86 & 2.00 \\
\hline 12 & 5.05 & 8.61 & 1.66 \\
\hline 13 & 3.55 & 8.61 & 1.57 \\
\hline 14 & 5.80 & 13.44 & 1.31 \\
\hline 15 & 3.99 & 9.02 & 2.20 \\
\hline 16 & 5.14 & 11.04 & 1.31 \\
\hline 17 & 3.41 & 8.83 & 1.86 \\
\hline 18 & 4.67 & 9.37 & 1.70 \\
\hline 19 & 3.67 & 8.74 & 1.17 \\
\hline 20 & 4.66 & 10.78 & 1.35 \\
\hline 21 & 4.70 & 11.52 & 1.11 \\
\hline 22 & 5.71 & 12.30 & 1.23 \\
\hline 23 & 5.48 & 9.86 & 1.32 \\
\hline 24 & 5.13 & 9.64 & 1.92 \\
\hline 25 & 4.96 & 13.76 & 1.61 \\
\hline 26 & 5.57 & 9.72 & 1.74 \\
\hline 27 & 4.13 & 10.21 & 1.40 \\
\hline 28 & 6.68 & 12.82 & 1.73 \\
\hline 29 & 6.44 & 10.80 & 1.61 \\
\hline 30 & 3.88 & 9.37 & 1.40 \\
\hline 31 & 3.35 & 8.60 & 1.85 \\
\hline 32 & 5.06 & 9.48 & 1.98 \\
\hline 33 & 5.34 & 10.87 & 1.84 \\
\hline 34 & 4.47 & 10.86 & 1.83 \\
\hline 35 & 4.66 & 11.49 & 2.67 \\
\hline 36 & 5.46 & 11.82 & 1.57 \\
\hline 37 & 5.19 & 12.95 & 1.46 \\
\hline 38 & 4.36 & 9.01 & 2.23 \\
\hline 39 & 2.95 & 7.67 & 1.40 \\
\hline 40 & 4.39 & 10.00 & 1.38 \\
\hline 41 & 3.95 & 10.21 & 1.38 \\
\hline 42 & 5.47 & 8.49 & 1.40 \\
\hline 43 & 4.22 & 8.94 & 2.11 \\
\hline 44 & 4.55 & 8.03 & 1.96 \\
\hline 45 & 3.33 & 7.49 & 1.84 \\
\hline
\end{tabular}

Table 4

Known and estimated values for brilliance and yield (models 4, 13 and 58), and respective errors

\begin{tabular}{lllllll}
\hline Model & $\begin{array}{l}\text { Brilliance } \\
(\text { known})\end{array}$ & $\begin{array}{l}\text { Brilliance } \\
\text { (estimated) }\end{array}$ & $\begin{array}{l}\text { Error } \\
(\%)\end{array}$ & $\begin{array}{l}\text { Yield } \\
(\text { known })\end{array}$ & $\begin{array}{l}\text { Yield } \\
(\text { estimated })\end{array}$ & $\begin{array}{l}\text { Error } \\
(\%)\end{array}$ \\
\hline $\mathbf{4}$ Eight \\
$\begin{array}{r}\text { round } \\
\mathbf{1 3 ~ C r o s s} \\
\text { point }\end{array}$ & 70.5 & 74.7 & 4.81 & 0.226 & 0.233 & 3.15 \\
$\mathbf{5 8 ~ S p i r o}$ & 86.9 & 71.4 & 0.98 & 0.246 & 0.266 & 8.03 \\
\hline
\end{tabular}

variation of the training performance error (the mean square error - mse) during 5000 epochs (presentation of 


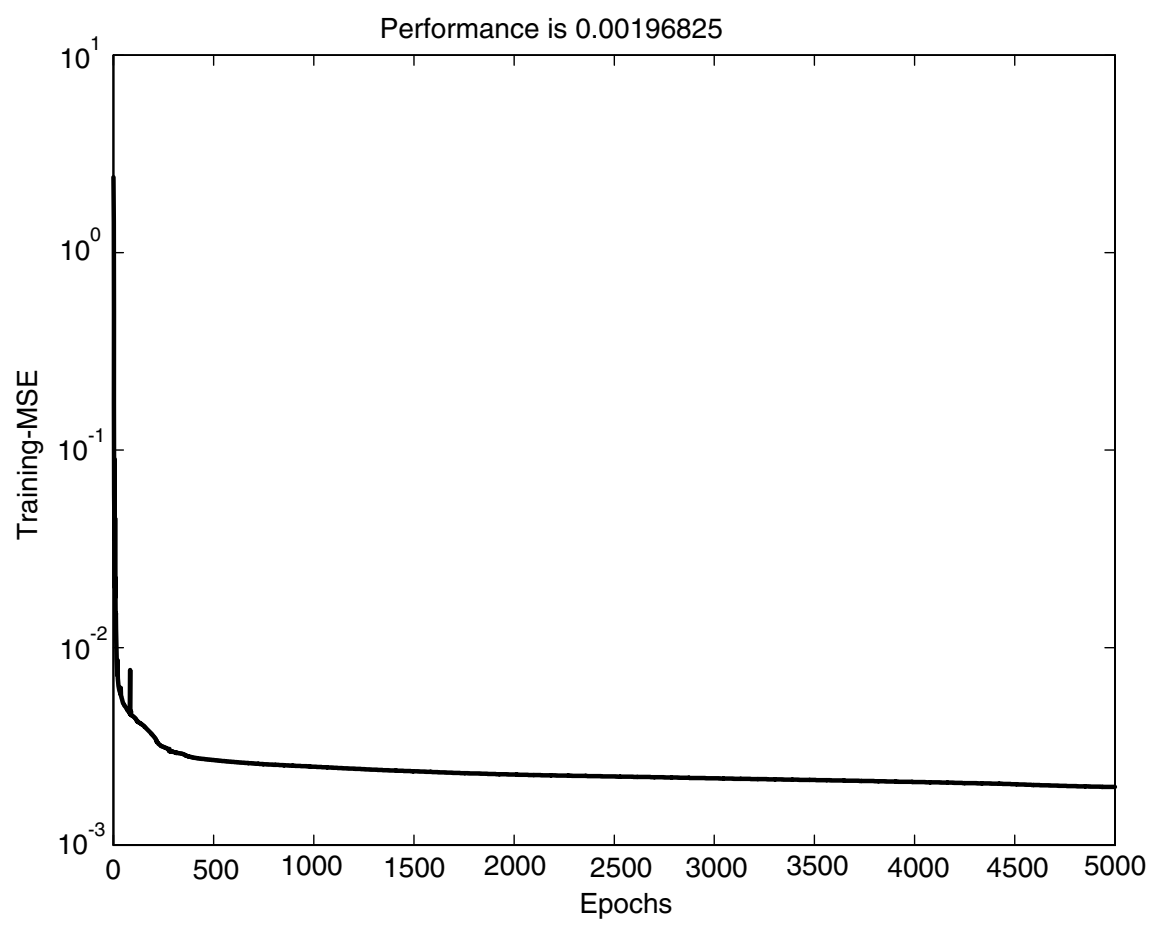

Fig. 10. Evolution of the performance index (mean square error of the ANN) during the training procedure.

the training data set) is shown in Fig. 10. Observe that the mse reach a value about $10^{-3}$. At the end of this learning procedure, the connections weight were obtained, representing the extracted knowledge of the studied problem.

For the following tests, a different strategy is considered: the called regularization method. This training strategy is applied to make the most of a limited supply of studied problem data, and the main goal is to improve the ANN generalization. The main training procedure difference concerns the performance function (usually the sum of squares of the network errors). The optimal performance function is obtained by adding a term that takes in account the mean of the sum of squares of the network weights and biases.

$\mathrm{mse}_{\mathrm{reg}}=\gamma \mathrm{mse}+(1-\gamma) \mathrm{msw}$

where $\gamma$ is the performance ratio, and

$\mathrm{msw}=\frac{1}{n} \sum_{j=1}^{n} w_{j}^{2}$

This training performance function allows the network to have smaller weights and biases, and the network response becomes smoother and less likely to overfit. An automated regularization, that can determine the optimal regularization parameters, based on Bayes' theorem, is proposed in [20]. In this automated procedure, the weights and biases of the network are considered as random variables with specified distributions. The parameters are related to the unknown variances associated with these distributions, and they can be estimated using statistical techniques.
The results of the training and test procedure using regularization can be seen in Table 5. These results present a strong convergence and repetition (without the results fluctuations seen in the precedent used strategy). The architecture considered here presents the input layer with six neurons, one hidden layer with six neurons, and the output layer with two neurons. This ANN structure was obtained using pruning methods $[18,19]$. For these tests, the average values of mean and maximum errors obtained are $4.05 \%$ and $11.40 \%$.

These results show that the ANN-based computational tool was capable of extracting knowledge from the prediction problem concerning evaluation parameters of faceting diagrams. The refinement of this CAD tool depends mainly of amassing a more representative and diversified sample of lapidary gemstone models, including an exhaustive array of shapes and proportions.

Considering the diversity of geometric possibilities of lapidary models, the limitations of the models data set used in the work, the physical characteristics of the problem, the important complexity of the relationship between the input

Table 5

Generalization method: known and estimated values for brilliance and yield (models 4, 13 and 58), and respective errors

\begin{tabular}{|c|c|c|c|c|c|c|}
\hline Model & $\begin{array}{l}\text { Brilliance } \\
\text { (known) }\end{array}$ & $\begin{array}{l}\text { Brilliance } \\
\text { (estimated) }\end{array}$ & $\begin{array}{l}\text { Error } \\
(\%)\end{array}$ & $\begin{array}{l}\text { Yield } \\
\text { (known) }\end{array}$ & $\begin{array}{l}\text { Yield } \\
\text { (estimated) }\end{array}$ & $\begin{array}{l}\text { Error } \\
(\%)\end{array}$ \\
\hline $\begin{array}{l}4 \text { Eight } \\
\text { round }\end{array}$ & 78.5 & 75.6 & 3.65 & 0.226 & 0.233 & 3.03 \\
\hline $\begin{array}{c}13 \text { Cross } \\
\text { point }\end{array}$ & 70.7 & 71.3 & 0.86 & 0.246 & 0.274 & 11.4 \\
\hline 58 Spiro & 86.9 & 86.5 & 0.49 & 0.239 & 0.227 & 4.84 \\
\hline
\end{tabular}


( $F, T / W, T / L, P / W, C / W, H / W)$ and the output (brilliance and yield) data, the obtained results show the proposed ANN architecture may be considered as a promising computational tool for lapidary development.

If additional data is made available, the proposed ANN based system can be easily retrained to capture additional features from the data, thus enhancing its classification performance.

\section{Conclusion}

This paper presented a general study of the problem of gem lapidary as a combination of physical and geometric effects influencing the evaluated beauty of faceted gems, through the light-material interactions, the material properties, and the polyhedral shapes. Considering the specific case of solid crystal quartz, an ANN-based design tool was proposed for the prediction of important evaluation properties (brilliance and yield).

The training and test procedures confirmed the work hypothesis about the exploitable adequacy of this class of artificial intelligence instrument, and it opens interesting possibilities for CAD tools development in the search for faceted gems design improvements, concerning an economic domain of indubitable relevance, specially for developing countries with important minerals resources, as the case of Brazil.

\section{Acknowledgement}

The authors would like to acknowledge the financial support of CNPq, FAPEMIG, FAPESP, and CAPES (Brazil).

\section{References}

[1] E.J. Gubelin, F.-X. Erni, Gemstones: Symbols of Beauty and Power, Geoscience Press, Tucson, 2000.

[2] R. Webster, Gems: Their Sources, Descriptions and Identification, vol. 2, Butterworth, London, 2002.

[3] W.E. Dengenhard, Gems and Gemology 14 (9) (1974) 259-270.

[4] K. Cai, J.T. Xia, et al., Computational Materials Science 34 (2) (2005) 166-172.

[5] P. Fermo, F. Cariati, et al., Applied Physics A: Materials Science and Processing 79 (2004) 299-307.

[6] A. Shahsavand, A. Ahmadpour, Computers and Chemical Engineering 29 (10) (2005) 2134-2143.

[7] T.S. Hemphill, I.M. Reinitz, et al., Gems and Gemology 34 (3) (1998) 58-183.

[8] K. Inoue, Journal of the Gemmological Society of Japan 20 (14) (1999) 153-167.

[9] I.M. Reinitz, M.L. Johnson, et al., Gems and Gemology 37 (3) (2001) 174-197.

[10] T. Holden, M. Serearuno, Journal of Intelligent Manufacturing 16 (1) (2005) 21-38.

[11] S. Guy, C. Soler, ACM Transactions on Graphics 23 (3) (2004) 231238.

[12] Brazilian Institute of Precious Gemstones and Metals, IBGM Website (in Portuguese). $<\mathrm{http}: / / w w w . i b g m . c o m . b r>$ (accession 2005).

[13] Centre for the Promotion of Imports from Developing Countries, EU Market Survey 2004: Jewellery, CBI, Rotterdam, 2004.

[14] J.R. Sauer, Brazil, Paradise of Gemstones, Amsterdam Sauer, Rio de Janeiro, 1982.

[15] J. Graham, Gram faceting website. <http://www.faceters.com> (accession 2004).

[16] J.M. Sasián, P. Yantzer, T. Tivol, Optics and Photonics News 14 (4) (2003) 24-31.

[17] A.K. Jain, J. Mao, K.M. Mohiuddin, IEEE Computer 29 (3) (1996) 56-63.

[18] S. Haykin, Neural Networks: A Comprehensive Foundation, Prentice Hall, New York, 1998.

[19] I.A. Basheer, M. Hajmeer, Journal of Microbiological Methods 43 (1) (2000) 3-31.

[20] D.J.C. MacKay, Neural Computation 4 (3) (1992) 415-447. 